\title{
Prevalence of nephropathy among type two diabetes patients in Eastern Mediterranean Region: A systematic review and meta-analysis
}

Shahryar Zeighami

Shiraz University of Medical Sciences

Sonia Shaabani

Shiraz University of Medical Sciences

Mohammad Moheb

Shiraz University of Medical Sciences

Maryam Pakfetrat

Shiraz University of Medical Sciences

Seyede Pegah Azarchehry

Tarbiat Modares University Faculty of Biological Sciences

Saeid Dehghankhalili

Shiraz University of Medical Sciences

Alireza Mirahmadizadeh

Shiraz University of Medical Sciences

Alireza Heiran ( $\square$ heiran.alireza@gmail.com )

Shiraz University of Medical Sciences https://orcid.org/0000-0001-6567-5306

\section{Research}

Keywords: Prevalence, Diabetes, Nephropathy, Eastern Mediterranean Region, Review

Posted Date: December 29th, 2021

DOl: https://doi.org/10.21203/rs.3.rs-518100/v2

License: (c) (i) This work is licensed under a Creative Commons Attribution 4.0 International License.

Read Full License 


\section{Abstract}

Objectives: The global epidemic of Diabetes Mellitus exerts a substantial burden on health services. This high prevalence of diabetes leads to an increase in diabetic complications, especially diabetic nephropathy, a leading cause of end stage renal disease and renal replacement therapies. Due to its' the high prevalence reported by Individual studies in EMR, we conducted a meta-analysis of relevant studies to estimate diabetic retinopathy in EMR.

Method: We searched for the relevant keywords in title and abstract of Medline/PubMed, Scopus, Embase, Web of knowledge, and Gray literature from Jan 1st, 2000 to December 13, 2019. The two-step screening was carried out by two independent researchers and relevant data were extracted. Estimation of summary proportions, subgroup analysis, meta-regressions, and publication bias assessment were performed.

Results: Out of 3,225 identified citations, 38 articles were entered into meta-analysis, involving 112,235 patients. The prevalence of nephropathy in type two diabetes was $26.34 \%(95 \%$ confidence interval $(\mathrm{Cl})=$ $21.04,32 \%)$; and it was $30.42 \%(95 \% \mathrm{Cl}=23.38,37.94 \%)$ in males and $22.1 \%(95 \% \mathrm{Cl}=16.05,28.79 \%)$ in females. The meta-regression analysis showed that HDI, publication year, mean duration of diabetes, mean age, and diagnostic test were not significant moderators $(p=.332, .725, .280, .220$, and .468 , respectively).

Conclusions: Diabetic nephropathy high prevalence in EMR implicates the importance of diabetes screening, periodic examinations, diabetes care, and risk factor controls. Large-scale longitudinal studies should be conducted in EMR.

\section{Introduction}

The epidemic of diabetes mellitus and its complications is a major public health concern that is rapidly growing in most of countries all around the world ${ }^{[1]}$. In 2000, the global prevalence of diabetes was estimated to be $2.8 \%$, about 171 million individuals in all age-group ${ }^{[2]}$. In 2017 , this number was reported as 425 million by the International Diabetes Federation (IDF), which will be projected to 642 million by $2040^{[3]}$.

Type two diabetes is a major leading cause for disability-adjusted life years (DALYs), accounting for 57.4 million DALYs in 2017 with a 34\% increase from $2007^{[4]}$. In kind, from the Global Burden of Disease 2015 study, DALYs of diabetes in EMR (Eastern Mediterranean Region) were 6.71 million, which were significantly higher than the expected rates based on Socio-demographic Index (SDI) in 2015 and higher than in all other WHO regions ${ }^{[5]}$.

According to IDF, the prevalence of diabetes mellitus in middle East and North Africa (MENA) was 9.1\% (35.4 million) in 2015 and $12.8 \%$ (55 million) in 2019, and it projects to $15.7 \%$ (108 million) in $2045^{[6]}$. All data indicate a high prevalence of diabetes mellitus in MENA, resulted from the changes in the 
socioeconomic conditions, urbanization, change in dietary habits, increase in sedentary lifestyle, causing marriage, etc.- as well as geopolitical instability, migration and demographic transition, and disparate distribution of resources ${ }^{[7]}$.

Hyperglycemia promotes to both microangiopathies and microangiopathies in patients with type two diabetes. Diabetic nephropathy is a frequent critical microvascular complication, which occurs in $20-40 \%$ of diabetic patient. The risk of developing diabetic nephropathy is low in a normoalbuminuric patient, but once microalbuminuria, the first clinical evidence of diabetic nephropathy, occurred, it can proceed to macroalbuminuria or overt nephropathy. In the presence of macroalbuminuria, glomerular filtration rate (GFR) falls, and as time goes by, end stage renal disease (ESRD) will happen ${ }^{[8-10]}$. Noticeably, though the prevalence of diabetes-induced ESRD is not clear in Asia, an increasing trend has been reported even in countries with low incidences of type two diabetes ${ }^{[11]}$.

Due to the high prevalence of type two diabetes, it is estimated that these patients include more than half of diabetics with ESRD requiring renal replacement therapies ${ }^{[12]}$, making diabetic nephropathy as the primary single cause of ESRD in the Western world (for example $44-45 \%$ in a U.S. survey in 2002) ${ }^{[13]}$. Therefore, to decrease burdens of diabetic nephropathy and ESRD on the health system and families' quality of life, the surveillance service should promote screening diabetics to yield early intervention as well as statistical information about the prevalence of diabetic nephropathy for the health care policy making. The prevalence of diabetes and its complications in EMR are high enough to become a public health concern ${ }^{[14,15]}$. EMR is a sub-community of WHO with a group of countries located in southwest Asia, Western Asia and North Africa, and all consider developing with heterogeneous economies ${ }^{[16,17]}$.

To the best of our knowledge, no study evaluated the prevalence of diabetic nephropathy in EMR. A systematic epidemiologic data is vital for governmental health legislation to early detection and prompt intervention. Therefore, we conducted a systematic review and meta-analysis of relevant studies published since 2000 to estimate nephropathy among type two diabetes patients in EMR.

\section{Methods}

\section{2-1- Search strategy}

In this systematic review and meta-analysis, we explored Medline/PubMed, Scopus, Embase, and Web of knowledge, as well as Google Scholar (the gray literature), January 2020, for studies on the prevalence of nephropathy in type two diabetes published from Jan $1^{\text {st }}, 2000$ to December 13, 2020 in EMRO. The keywords list is booked in the appendix 1 . Also, reference lists of review studies were manually searched. Then, identified articles collected in endnote-x9. Our study was not registered, but its protocol is documented in library of Shiraz University of Medical Sciences.

\section{2-2-Selection criteria}


In first step, titles and abstracts of article were screened by two independent researchers (S.S and M.M). In second step, qualified articles were re-screened through investigating the whole manuscript by the same researchers. An article was included if it studied the prevalence of nephropathy amongst patients with type two diabetes in a normal population. In case of conflict, the first author (S.Z) made the final decision whether to include or exclude an article. The JBL (Joanna Briggs Institute) checklist for possibility of bias in its design, conduct and analysis in systematic reviews was used for methodological quality assessment of included studies ${ }^{[\mathrm{S} 1]}$. The result of quality assessment is shown in Appendix 2.

\section{2-3- Data extraction}

All targeted statistics were entered into a checklist prepared as a spreadsheet. This check list included: first author's name, publication year, recruitment time span, country, number of sample size (total, female, male), proportion (\%) and upper and lower $95 \%$ confidence interval (Cl) of nephropathy (total, female, male), participants' age range, mean duration of type two diabetes, and diagnosis test.

\section{2-4- Statistical analysis}

Statistical analysis was carried out by R program (v3.4.1) ${ }^{[\mathrm{S} 2]}$ using metafor ${ }^{[\mathrm{S} 3]}$ and meta ${ }^{[\mathrm{S} 4]}$ packages.

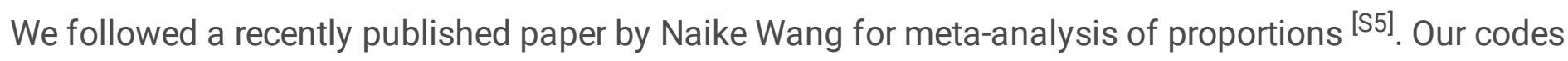
are provided in appendix 3 .

Summary effect size was calculated by the random-effects model since this is expected that both between-study variance ( $\mathrm{tau}^{2}$; true effect sizes or real variation related to populations' characteristics) and within-study variance (due to the random sampling error) exist in a series of observational studies. Furthermore, the random-effects model generates more generalizable results. The random-effects model was carried out by the restricted maximum likelihood method (REML). Moreover, we applied the doublearcsine transformation method to transform proportions into the normal distribution to obtain a more accurate estimate of summery proportion.

Since a considerable variation (heterogeneity) usually exists around the summary proportion, we visually inspected the output forest plot for non-overlapping $95 \% \mathrm{Cl}$ studies around the summary effect), performed $X^{2}$ test (general heterogeneity), and calculated $R$ statistic (the proportion of heterogeneity related to the between-study variance). It is worth noting that our estimated $R$ was $99.69 \%(95 \% \mathrm{Cl}=$ $99.52,99.81)$, denoted that approximately all heterogeneity could be refer to the between-study variance; hence, we carried out sub-group analyses and meta-regressions to explore different potential moderators of this heterogeneity of the effect sizes, including HDI (Human Development Index; a relative measure of the living standards in human societies ${ }^{[56]}$ ), publication year, diagnostic test, mean duration of diagnosed type two diabetes, and mean age. For sub-group analysis, the random-effects model was used to obtain summary effect sizes within each subgroup and then fixed-effect model was used to test whether these effects differed significantly from each other. 
To visualize studies' effects and their confidence intervals, we yielded a forest plot. It is worth noting that by visual inspection of another forest plot (appendix 4) that sorted the studies according to their precisions (using standard error (SE)), 8 out of 12 studies with more than 1000 sample size were considered as outlier, which confirmed the high overall heterogeneity. However, we recruited a quantitative test to obtain truly outliers. It was carried out by externally standardized residuals; that is, a study with absolute value larger than 3 might be and outlier. Also, leave-one-out estimates for the amount of heterogeneity was carried out, which considers a study as an outlier if its exclusion leads to a considerable influence on the summary proportion. In externally standardized residuals test, we could not find any study with absolute value of larger than 3 (appendix 5). Moreover, leave-one-out diagnostic test did not find any influential outlier (appendix 6-9).

We yielded funnel plot and carried out objective tests, the egger regression test, for publication bias. However, it should be noticed that in epidemiology studies, papers reporting either low proportions or high proportions are likely to be published. Therefore, exploring the publication bias might not be applicable or informative in meta-analyses of observational studies ${ }^{[57]}$.

\section{Results}

\section{3-1- Results of the search}

We initially identified 3,325 citations. After discarding duplicates and publications before 2000, 2,936 studies remained for title and abstract screening, which resulted in 66 articles for second round of screening. In addition, 7 studies were manually added. After reading 73 full texts, 35 papers discarded and 38 articles remained entered into the meta-analysis. The reasons for excluding that 35 articles were: studies out of EMRO $(n=4)$, reporting incidence of nephropathy $(n=2)$, review or meta-analysis study ( $=5)$, non-English papers $(n=5)$, full text unavailable $(n=5)$, and unclear results or irrelevant papers $(n=$ 14). The flowchart of data extraction is shown in figure 1 and basic characteristics of the included studies in meta-analysis is shown in table 1. Our dataset consists of 38 studies that were published from 2000 to 2019 and contained data on the population-based prevalence of nephropathy in type two diabetes in EMRO. The sample sizes of the included studies ranged from 99 to 54,670 patients, with a cumulative size of 112,235 patients.

\section{3-2- Results of the meta-analysis}

\section{3-2-1- Heterogeneity}

The output of heterogeneity analysis showed that $\operatorname{tau}^{2}$ was $0.04(95 \% \mathrm{Cl}=0.02,0.06), P$ was $99.69 \%(95 \%$ $\mathrm{Cl}=99.52,99.81)$, and the Q-statistic was 9,418.874 ( $p<0.0001)$, which all suggested a high heterogeneity in the effect sizes. Also, the high value of $P$ indicated that almost all heterogeneity could be due to the between-study variance (fig. 2).

\section{3-2-2- Prevalence of nephropathy in type two diabetes}


We found that the summary proportion was 0.2634 ( $95 \% \mathrm{Cl}=0.2104,0.3200)$, which represented $26.34 \%$ prevalence of nephropathy in type two diabetes (fig. 2). Also, by performing separate meta-analyses in male and female datasets, the prevalence of diabetic nephropathy was 30.42\% [0.3042 (95\% Cl= 0.2338 , $0.3794)]$ in males (fig. 3) and $22.10 \%[0.2210(95 \% \mathrm{Cl}=0.1605,0.2879)]$ in females (fig. 4).

\section{3-2-3- Prevalence of nephropathy in diabetes based on sub-group analysis by HDI}

In sub-group analysis by $\mathrm{HDI}$, the summary effect proportions were $0.2117(95 \% \mathrm{Cl}=0.0110,0.562)$, 0.2618 (95\% Cl= 0.1777, 0.3557), 0.2039 (95\% Cl= 0.1134, 0.3127), and 0.3093 (95\% Cl= 0.2216, 0.4044) for the four subgroups of low, medium, high, and very high, respectively. Low HDI countries and very high HDI countries had the lowest (21.17\%) and the highest (30.93\%) nephropathy prevalence in type two diabetes.

As a nature of our analysis (separate random effects models in each subgroup), within-group estimates of tau $^{2}$ were 0.105 [Q(df = 2) = 426.085, $\left.p<0.001\right], 0.032$ [Q $\left.(\mathrm{df}=11)=2723.9, p<0.001\right], 0.031$ [Q(df = 7)= $350.631, p<0.001]$, and 0.038 [Q $(\mathrm{df}=14)=2912.522, p<0.001]$, for sub-groups low, medium, high, and very high, respectively. We did not find a significant difference between the four subgroup summary estimates $(Q(d f=3)=2.325, p=0.508)$, representing that HDI did not share on the true heterogeneity of the summary proportion.

\section{3-3- Results of the Meta-regression}

The meta-regression analysis was performed for five different variables, include HDI, publication year, mean duration of diagnosed diabetes, diagnostic test, and mean age.

The slope of the estimated regression line for all of the five different variables were near to horizontal, which did not suggested significant moderating effects on the prevalence of nephropathy in type two diabetes $([\mathrm{HDI}] \mathrm{Q}(\mathrm{df}=1)=0.9398$, coefficient $=0.0297, Z=0.9695, p=0.332$; [publication year] $\mathrm{Q}(\mathrm{df}=1)=$ 0.124 , coefficient $=-0.002, Z=-0.3522, p=0.725$; [mean duration of diagnosed diabetes] $\mathrm{Q}(\mathrm{df}=1)=1.166$, coefficient $=0.0095, Z=1.0798, p=0.280$; [mean age] $\mathrm{Q}(\mathrm{df}=1)=1.5048$, coefficient: $0.0088, Z=1.2267$, $p=0.220$; [diagnostic test] $\mathrm{Q}(\mathrm{df}=1)=0.5268$, coefficient: $0.0625, Z=0.7258, p=0.468)$ (fig. 5).

Importantly, in all meta-regression plots, most of the studies were outside the $95 \% \mathrm{Cl}$ boundaries, which implied on the presence of unknown or missed parameters affecting the prevalence of nephropathy in type two diabetes. A zero value of $\mathrm{R}^{2}$, which represents the amount of between-study heterogeneity explained by a modulator, supports it.

\section{3-4- Publication bias}

Visual inspection of the funnel plot showed asymmetry in our data (fig. 6). Also, the Egger test shows that the funnel plot was significantly asymmetrical $(Z=2.0983, p=0.0359)$. Furthermore, the funnel plot of proportions against sample sizes showed that small-study effect was present in our meta-analysis (appendix 10). Importantly, it should be noticed that the just mentioned asymmetry might not necessarily 
indicate publication bias; that is, other parameters that interfere with the inclusion of small studies may contribute to this asymmetry in observational studies ${ }^{[7]}$. First, we previously showed a high betweenstudy heterogeneity. Again, a substantial number of studies fall out of the two limit lines of in forest plot, which confirmed the high heterogeneity. In other words, this high between-study heterogeneity might be due to the particular reasons. Second, we excluded foreign languages' small studies, the so-called English language bias. Finally, irrespective of the sensitive search strategy, gray literature search and manual search in references for relevant studies, the citation bias might be happened.

\section{Discussion}

Given the massive costs of renal replacement therapies and the projecting incidence of ESRD, using available evidence is demanded to adopt better programs and strategies to answer the barriers in receiving high-quality care and improving quality of life ${ }^{[18]}$. Since there is a paucity of country-specific epidemiology data in the region, we reviewed 38 articles in EMR studied the prevalence of nephropathy as a microvascular complication of type two diabetes.

On the basis of the data from 38 studies and approximately 110,000 participants with diabetes, the main finding of our study was the prevalence of $26 \%$ for nephropathy among patients with type 2 diabetes in EMR. In UKPDS (U.K. Prospective Diabetes Study) ${ }^{[19]}$ and the "Pima Indian population" ${ }^{[20]}$ cohorts on type two diabetes subjects and another study ${ }^{[21]}$ on 32,208 type 2 diabetes patients from 33 countries, the prevalence of microalbuminuria was $38,50 \%$ and $38.8 \%$ respectively. It should be noticed that the second cohort had been conducted prior to widespread use of RAAS inhibitors. Considering other studies, with a varying range of microalbuminuria $(26.9-31.6 \%)^{[22,23]}$, this heterogeneity may be resulted from different genetic background, lifestyle and awareness of kidney complications of diabetes ${ }^{[24-26]}$. Furthermore, we showed that rate was $30 \%$ in male and $22 \%$ in female. On the contrary, several community-based studies reported that diabetic nephropathy affects males and females equally in most regions ${ }^{[27]}$, except a male predominance in Southern Sub-Saharan Africa and a female predominance in Tropical Latin America ${ }^{[28]}$. This is hypothesized that better glomerular filtration rate in men than women, (so overestimating rate of nephropathy in women), hormonal differences, and social realm norms (i.e., tendency to perform screening) may tend females to develop diabetic nephropathy ${ }^{[29]}$. However, gender might be rather an adjustment variable in studies conducted on diabetic nephropathy, and any gender predominance it is thought to be incidental ${ }^{[30]}$.

Similar to our analysis, the "Pima Indian population" [20] cohort and another study on U.S. population showed that the prevalence trend remained fairly stable during the past two decades ${ }^{[11,20]}$ which is probably a worldwide status ${ }^{[31]}$, irrespective of an increase in diabetes prevalence over time, especially in low- and middle-income countries. While taking caution with respect to inadequate publications and undiagnosed cases, this stable microvascular complication of type two diabetes prevalence might reflect success in preventing type two diabetes nephropathy but little success in preventing incident type two diabetes. Also, this is plausible that the consequence of various opposing factors has ultimately kept the 
nephropathy rate constant in EMR. For example, although many "Persian Gulf" countries have made progression in recent years and developed their health system, still the rate of diabetes is high due to the many fundamental reasons that account for as risk factors of diabetic nephropathy too. Also, several EMR countries have encountered serious economic and political issues because of warfare, sanction, and refugee, which drastically have compromised the health care system.

The secondary outcome of our analysis showed that low HDI countries had the lowest (21.2\%) and very high HDI countries had the highest (30.9\%) prevalence of nephropathy among type two diabetes patients. It implies on the importance of health surveillance system, for example extensive screening methods in in countries with very-high HDI compared to countries with low HDI. In addition, we have learnt that some the metabolic syndrome components are strong risk factors of microalbuminuria amongst subjects with type two diabetes. Currently, EMR suffers from the epidemics of metabolic syndrome, especially in Persian Gulf countries which are the very-high-HDI countries of EMR. Taking these into account it may partially explain the heterogeneity of prevalence in countries with different HDI. By and large, perhaps early detection of diabetes and early-stage nephropathy, establishing pre-ESRD renal registries, early treatment, detecting untreated subjects as well as continuity of care, glycemic control and reducing modifiable risk factors to slow down the progression of nephropathy, life style modification and weight control, and patients' education on their disease and the importance of annual checkup, follow up protocol at asymptomatic phase, family support, etc. are the most important health system's interventions to reduce the burden of this disease ${ }^{[32-34]}$. This statement can be supported by a review that showed more than half of subjects resided in MENA region did not meet recommended care targets ${ }^{[35]}$.

Since a high between-study heterogeneity is expected in meta-analysis of cross-sectional studies, we performed meta-regressions; however, we did not find any significant association between the prevalence and different moderators (HDI, publication year as a proxy of year, mean duration of diabetes, diagnostic test, and mean age). We believe this is agreed with available evidences. For example, though diabetic nephropathy is expected to be higher amongst elder subjects with diabetes type 2 who have lived longer with it, the role of age in the development of diabetic nephropathy is unclear [36]. In our analysis, the prevalence of nephropathy was directly increased by age and duration of living with type two diabetes, but the slopes of both moderators were not statistically significant. It suggests that the variation in the prevalence of diabetic nephropathy depends on other factors. Retnakaran et al. ${ }^{[19]}$ mentioned that development of albuminuria is associated with increased baseline systolic blood pressure, male sex, increased waist circumference, positive smoking history, and high triglyceride/HDL ratio. Some of these risk factors are linked to insulin resistance and metabolic syndrome, which may not be matched in different populations. Furthermore, it should be kept in mind that this heterogeneity in the prevalence of nephropathy can be also attributed to differences in study design, setting and quality; however, However, the possibility of true ethnic differences cannot be neglected.

\section{Conclusion}


Our study yielded the first pooled analysis to estimate the prevalence of diabetic nephropathy in EMR. Based on our analysis this estimation was $26 \%$. Our result implicates the importance of early diabetes and pre-ESRD screening and diagnosis, early intervention, and glycemic and risk factor control. Largescale longitudinal studies are warranted in most EMR countries.

\section{Declarations}

Competing interests: The Authors declare that there is no competing interest.

Funding: The present article was financially supported by the Shiraz University of Medical Sciences [grant number 19294-109-01-97].

Author contributions: All authors conceived the study and were responsible for designing the protocol. SZ, AH, MP, AM designed the study. AH, SPA and SD did the literature search. SS and MM selected the studies and extracted the relevant information. All authors synthesized the data. SS, MM, SPA, and SD wrote the first draft of the paper. SZ, AM, MM, and AH provided critical guidance on the analysis and overall direction of the study. All authors critically revised successive drafts of the paper and approved the final version.

Availability of data and materials: The dataset supporting the conclusions of this article (data extracted from included studies and data used for all analyses) is available upon request to the corresponding author, Alireza Heiran. The R analytic codes are included within the article's additional file.

\section{References}

1. Saeedi P, Petersohn I, Salpea P, Malanda B, Karuranga S, Unwin N, et al. Global and regional diabetes prevalence estimates for 2019 and projections for 2030 and 2045: Results from the International Diabetes Federation Diabetes Atlas, 9th edition. Diabetes Res Clin Pract. 2019;157:107843.

2. Wild S, Roglic G, Green A, Sicree R, King H. Global prevalence of diabetes: estimates for the year 2000 and projections for 2030. Diabetes Care. 2004;27(5):1047-53.

3. Cho NH, Shaw JE, Karuranga S, Huang Y, da Rocha Fernandes JD, Ohlrogge AW, et al. IDF Diabetes Atlas: Global estimates of diabetes prevalence for 2017 and projections for 2045. Diabetes Res Clin Pract. 2018;138:271-81.

4. GBD 2017 DALYs and HALE Collaborators. Global, regional, and national disability-adjusted life-years (DALYs) for 359 diseases and injuries and healthy life expectancy (HALE) for 195 countries and territories, 1990-2017: a systematic analysis for the Global Burden of Disease Study 2017. Lancet. 2018;392(10159):1859-922.

5. GBD 2015 Eastern Mediterranean Region Diabetes and Chronic Kidney Disease Collaborators. Diabetes mellitus and chronic kidney disease in the Eastern Mediterranean Region: findings from the Global Burden of Disease 2015 study. Int J Public Health. 2018;63(Suppl 1):177-86. 
6. International Diabetes Federation. IDF Diabetes Atlas, 9th edn. Brussels, Belgium: 2019. Available from: https://www.diabetesatlas.org

7. Alhyas L, McKay A, Majeed A. Prevalence of type 2 diabetes in the States of the co-operation council for the Arab States of the Gulf: a systematic review. PLoS One. 2012;7(8):e40948.

8. Koye DN, Magliano DJ, Nelson RG, Pavkov ME. The Global Epidemiology of Diabetes and Kidney Disease. Adv Chronic Kidney Dis. 2018;25(2):121-32.

9. Reutens AT, Atkins RC. Epidemiology of diabetic nephropathy. Contrib Nephrol. 2011;170:1-7.

10. Wu AY, Kong NC, de Leon FA, Pan CY, Tai TY, Yeung VT, et al. An alarmingly high prevalence of diabetic nephropathy in Asian type 2 diabetic patients: the MicroAlbuminuria Prevalence (MAP) Study. Diabetologia. 2005;48(1):17-26.

11. de Boer IH, Rue TC, Hall YN, Heagerty PJ, Weiss NS, Himmelfarb J. Temporal trends in the prevalence of diabetic kidney disease in the United States. JAMA. 2011;305(24):2532-9.

12. American Diabetes Association. Nephropathy in Diabetes. Diabetes Care. 2004 (suppl 1);27:S79-83.

13. Rabkin R. Diabetic nephropathy. Clin Cornerstone. 2003;5(2):1-11.

14. Hajar S, Al Hazmi A, Wasli M, Mousa A, Rabiu M. Prevalence and causes of blindness and diabetic retinopathy in Southern Saudi Arabia. Saudi Med J. 2015;36(4):449-55.

15. Rabiu MM, Al Bdour MD, Abu Ameerh MA, Jadoon MZ. Prevalence of blindness and diabetic retinopathy in northern Jordan. Eur J Ophthalmol. 2015;25(4):320-7.

16. WorldHealthOrganization. VIH/SIDA differences entre hommes et femmes dans la Region de la Mediterranee orientale. 2005. Available from: https://apps.who.int/iris/handle/10665/116482

17. Kuper H, Polack S, Limburg H. Rapid assessment of avoidable blindness. Community Eye Health. 2006;19(60):68-9.

18. Reilly R, Evans K, Gomersall J, Gorham G, Peters MD, Warren S, et al. Effectiveness, cost effectiveness, acceptability and implementation barriers/enablers of chronic kidney disease management programs for Indigenous people in Australia, New Zealand and Canada: a systematic review of mixed evidence. BMC Health Serv Res. 2016;16:119.

19. Retnakaran R, Cull CA, Thorne KI, Adler Al, Holman RR; UKPDS Study Group. Risk factors for renal dysfunction in type 2 diabetes: U.K. Prospective Diabetes Study 74. Diabetes. 2006;55(6):1832-9.

20. Pavkov ME, Knowler WC, Bennett PH, Looker HC, Krakoff J, Nelson RG. Increasing incidence of proteinuria and declining incidence of end-stage renal disease in diabetic Pima Indians. Kidney Int. 2006;70(10):1840-6.

21. Parving HH, Lewis JB, Ravid M, Remuzzi G, Hunsicker LG; DEMAND investigators. Prevalence and risk factors for microalbuminuria in a referred cohort of type II diabetic patients: a global perspective. Kidney Int. 2006;69(11):2057-63.

22. Unnikrishnan RI, Rema M, Pradeepa R, Deepa M, Shanthirani CS, Deepa R, et al. Prevalence and risk factors of diabetic nephropathy in an urban South Indian population: the Chennai Urban Rural Epidemiology Study (CURES 45). Diabetes Care. 2007;30(8):2019-24. 
23. Yokoyama H, Sone H, Oishi M, Kawai K, Fukumoto Y, Kobayashi M. Japan Diabetes Clinical Data Management Study Group. Prevalence of albuminuria and renal insufficiency and associated clinical factors in type 2 diabetes: the Japan Diabetes Clinical Data Management study (JDDM15). Nephrol Dial Transplant. 2009;24(4);1212-9.

24. Lightstone L, Rees AJ, Tomson C, Walls J, Winearls CG, Feehally J. High incidence of end-stage renal disease in Indo-Asians in the UK. QJM. 1995;88(3):191-5.

25. Barbour SJ, Er L, Djurdjev O, Karim M, Levin A. Differences in progression of CKD and mortality amongst Caucasian, Oriental Asian and South Asian CKD patients. Nephrol Dial Transplant. 2010; 25(11):3663-72.

26. Wilkinson E, Randhawa G, Farrington K, Greenwood R, Feehally J, Choi P, Lightstone L. Lack of awareness of kidney complications despite familiarity with diabetes: a multi-ethnic qualitative study. J Ren Care. 2011;37(1):2-11.

27. Pavkov ME, Bennett PH, Knowler WC, Krakoff J, Sievers ML, Nelson RG. Effect of youth-onset type 2 diabetes mellitus on incidence of end-stage renal disease and mortality in young and middle-aged Pima Indians. JAMA. 2006;296(4):421-6.

28. Thomas B. The Global Burden of Diabetic Kidney Disease: Time Trends and Gender Gaps. Curr Diab Rep. 2019;19(4):18.

29. Carrero JJ, Hecking M, Ulasi I, Sola L, Thomas B. Chronic kidney disease, gender, and access to care: a global perspective. Semin Nephrol. 2017;37(3):296308.

30. Cobo G, Hecking M, Port FK, Exner I, Lindholm B, Stenvinkel P, et al. Sex and gender differences in chronic kidney disease: progression to end-stage renal disease and haemodialysis. Clin Sci. 2016;130(14):1147-63.

31. Thomas B. The Global Burden of Diabetic Kidney Disease: Time Trends and Gender Gaps. Curr Diab Rep. 2019;19(4):18.

32. Lo C, Teede H, Fulcher G, Gallagher M, Kerr PG, Ranasinha S, et al. Gaps and barriers in health-care provision for co-morbid diabetes and chronic kidney disease: a cross-sectional study. BMC Nephrol. 2017;18(1):80.

33. Lo C, Teede H, Ilic D, Russell G, Murphy K, Usherwood T, et al. Identifying health service barriers in the management of co-morbid diabetes and chronic kidney disease in primary care: a mixed-methods exploration. Fam Pract. 2016;33(5):492-7.

34. Thomas B. The Global Burden of Diabetic Kidney Disease: Time Trends and Gender Gaps. Curr Diab Rep. 2019;19(4):18.

35. Zabetian A, Kelli HM, Echouffo-Tcheugui JB, Narayan KM, Ali MK. Diabetes in the Middle East and North Africa. Diabetes Res Clin Pract. 2013;101(2):106-22.

36. Pavkov ME, Bennett PH, Knowler WC, Krakoff J, Sievers ML, Nelson RG. Effect of youth-onset type 2 diabetes mellitus on incidence of end-stage renal disease and mortality in young and middle-aged Pima Indians. JAMA. 2006.296(4):421-6. 


\section{Tables}

Page 12/21 
Table 1. Basic characteristics of the studies included in the review

\begin{tabular}{|c|c|c|c|c|c|c|c|}
\hline \# & Author, Year, HDI & $\begin{array}{l}\text { Time } \\
\text { period }\end{array}$ & $\mathrm{N}$ & $\mathrm{p}(\%)$ & Age (year) & $\begin{array}{c}\text { Mean } \\
\text { duration }\end{array}$ & $\begin{array}{c}\text { Diagnosis } \\
\text { tool }\end{array}$ \\
\hline 1 & Shera, 2004, M, Pakistan [S8] & NA & 500 & 20.2 & $55.2 \pm 10.6$ & $7 \pm \mathrm{SD} ?$ & NA \\
\hline 2 & $\begin{array}{l}\text { Al-Homrany, 2004, VH, Saudi } \\
\text { Arabia [S9] }\end{array}$ & NA & 208 & 54.3 & $56.2 \pm 8.8$ & $9.6 \pm 4.7$ & C3 \\
\hline 3 & Taleb, 2008, H, Lebanon ${ }^{[\mathrm{S} 10]}$ & $\begin{array}{l}2005- \\
2007\end{array}$ & 220 & 46.3 & $57.7 \pm 10.6$ & $8.2 \pm 6.6$ & C3 \\
\hline 4 & Zirie, 2003, VH, Qatar ${ }^{[\mathrm{S} 11]}$ & $\begin{array}{l}2001- \\
2002\end{array}$ & 225 & 40.9 & NA & NA & NA \\
\hline 5 & $\begin{array}{l}\text { Ghandour, 2018, H, } \\
\text { Palestinian }{ }^{[\mathrm{S} 12]}\end{array}$ & 2012 & 486 & 51.4 & $58 \pm 9.8$ & $9 \pm 7.5$ & $\mathrm{C} 1,2$ \\
\hline 6 & Ahmad, 2017, M, Pakistan ${ }^{[\mathrm{S} 13]}$ & $\begin{array}{l}2014- \\
2016\end{array}$ & 1280 & 31.56 & $54.9 \pm 11.6$ & $9.7 \pm 7.8$ & C1 \\
\hline 7 & Ali, 2013, M, Pakistan ${ }^{[\mathrm{S} 14]}$ & $\begin{array}{l}2011- \\
2012\end{array}$ & 113 & 44.2 & NA (30-70) & 0.5 & C3 \\
\hline 8 & Alrawahi, 2012, VH, Oman ${ }^{[\mathrm{S} 15]}$ & $\begin{array}{l}2010- \\
2011\end{array}$ & 699 & 42.5 & $51.86 \pm 11.75$ & NA & $\mathrm{C} 1$ \\
\hline 9 & $\begin{array}{l}\text { Al-Rubeaan, 2014, VH, Saudi } \\
\text { Arabia [S16] }\end{array}$ & NA & 54670 & 10.8 & $59.91 \pm 12.72$ & $13.55 \pm 8.14$ & $\mathrm{C} 3$ \\
\hline 10 & Al-Salman, 2009, VH, Bahrain [S17] & 2006 & 702 & 27.9 & $51.2 \pm 10.1$ & $8.6 \pm 6.2$ & C1 \\
\hline 11 & Cheema, 2018, VH, Qatar [S18] & $\begin{array}{l}2013- \\
2015\end{array}$ & 1034 & 15.3 & $55 \pm 10$ & $12.4 \pm 8.9$ & NA \\
\hline 12 & Abro, 2018, M, Pakistan ${ }^{[\mathrm{S} 19]}$ & $\begin{array}{l}2005- \\
2016\end{array}$ & 28601 & 31 & $52.5 \pm 11.3$ & $8.2 \pm 7.5$ & $\begin{array}{c}\mathrm{C} 3,4 \\
\text { (dipstick) }\end{array}$ \\
\hline 13 & $\begin{array}{l}\text { Afkhami-Ardekani, 2008, H, } \\
\text { Iran [S20] }\end{array}$ & $\begin{array}{l}2005- \\
2007\end{array}$ & 288 & 14.2 & $53.2 \pm 9.9$ & $9.3 \pm 6.3$ & C3 \\
\hline 14 & Esteghamati, 2008, H, Iran ${ }^{[S 21]}$ & $\begin{array}{l}2005- \\
2008\end{array}$ & 833 & 29.1 & $54.6 \pm 10.3$ & $8.3 \pm 6.4$ & $\mathrm{C} 3$ \\
\hline 15 & $\begin{array}{l}\text { Al-Halaweh, 2017, H, } \\
\text { Palestinian }{ }^{[\mathrm{S} 22]}\end{array}$ & 2015 & 1308 & 4.9 & $57 \pm 8.7$ & $7.1 \pm 6.25$ & $\begin{array}{c}\mathrm{C} 4 \\
(\mathrm{Cr}>1.4)\end{array}$ \\
\hline 16 & $\begin{array}{l}\text { Ahmed, 2017, VH, Saudi } \\
\text { Arabia }\end{array}$ & NA & 316 & 32.3 & $58 \pm 10$ & NA & $\begin{array}{c}\mathrm{C} 2,4 \\
\text { (micral) }\end{array}$ \\
\hline 17 & Mansour, 2009, M, Iraq ${ }^{[S 24]}$ & $\begin{array}{l}2003- \\
2009\end{array}$ & 4926 & 6.6 & $55 \pm 13.1$ & $7.7 \pm 6.7$ & $\begin{array}{c}\mathrm{C} 4 \\
\text { (dipstick) }\end{array}$ \\
\hline 18 & Jelinek, 2017, VH, UAE ${ }^{[S 25]}$ & $\begin{array}{l}2014- \\
2015\end{array}$ & 490 & 32.85 & $60.6 \pm 11.3$ & NA & $\mathrm{C} 3$ \\
\hline 19 & Al-Maskari, 2008, VH, UAE ${ }^{[S 26]}$ & $\begin{array}{l}2003- \\
2004\end{array}$ & 451 & 61.2 & $53 \pm 13$ & NA & $\begin{array}{c}\mathrm{C} 4 \\
\text { (micral) }\end{array}$ \\
\hline
\end{tabular}




\begin{tabular}{|c|c|c|c|c|c|c|c|}
\hline 20 & Akhter, 2017, M, Pakistan [S27] & $\begin{array}{l}2014- \\
2015\end{array}$ & 876 & 13.8 & $53.1 \pm 11.9$ & $7.6 \pm 7.1$ & NA \\
\hline 21 & Uddin, 2018, M, Pakistan [S28] & $\begin{array}{l}2015- \\
2016\end{array}$ & 891 & 24.4 & $47.7 \pm 10.6$ & 0.5 & $\mathrm{C} 1$ \\
\hline 22 & Bamashmoos, 2013, L, Yemen ${ }^{[S 29]}$ & $\begin{array}{l}2011- \\
2012\end{array}$ & 500 & 33.6 & $56.3 \pm 32$ & $10.6 \pm 5.4$ & $\mathrm{C} 3$ \\
\hline 23 & $\begin{array}{l}\text { Alaboud, 2016, VH, Saudi } \\
\text { Arabia [S30] }\end{array}$ & 2014 & 748 & 15 & $57.9 \pm 11.6$ & NA & NA \\
\hline 24 & $\begin{array}{l}\text { Alwakeel, 2011, VH, Saudi } \\
\text { Arabia [S31] }\end{array}$ & $\begin{array}{l}1989- \\
2004\end{array}$ & 1952 & 31.8 & $66.9 \pm 11.4$ & $15.4 \pm 7.5$ & $\begin{array}{c}\mathrm{C} 2,4 \\
\text { (dipstick) }\end{array}$ \\
\hline 25 & $\begin{array}{l}\text { Alramadan, 2019, VH, Saudi } \\
\text { Arabia [S32] }\end{array}$ & 2017 & 1111 & 14.5 & $57.6 \pm 11.1$ & $13.9 \pm 8.4$ & NA \\
\hline 26 & Hussein, 2019, L, Sudan [S33] & 2018 & 209 & 38.8 & $55.42 \pm 16.46$ & $8.04 \pm 6.98$ & $\mathrm{C} 1$ \\
\hline 27 & Ahmedani, 2005, M, Pakistan [S34] & 2003 & 2186 & 34 & $53.1 \pm 11.9$ & $8.8 \pm 5.21$ & $\begin{array}{c}\mathrm{C} 4 \\
\text { (micral) }\end{array}$ \\
\hline 28 & Shahwan, 2019, H, Palestine [S35] & $\begin{array}{l}2017- \\
2018\end{array}$ & 550 & 38.5 & $58.7 \pm 8.3$ & $8.9 \pm 6.8$ & C3 \\
\hline 29 & Ahmadani, 2008, M, Pakistan [S36] & NA & 99 & 33.3 & $53.12 \pm 10.66$ & $9.32 \pm 6.87$ & $\begin{array}{c}\mathrm{C} 4 \\
\text { (micral) }\end{array}$ \\
\hline 30 & Modesti, 2013, L, Yemen ${ }^{[S 37]}$ & NA & 1216 & 2.14 & NA (15-69) & NA & $\begin{array}{c}\mathrm{C} 4 \\
\text { (dipstick) }\end{array}$ \\
\hline 31 & Bener, 2014, VH, Qatar [S38] & $\begin{array}{l}2011- \\
2013\end{array}$ & 1633 & 12.4 & $45.3 \pm 15$ & $7.3 \pm 4.9$ & NA \\
\hline 32 & Khawaja, 2018, H, Jordan ${ }^{[\mathrm{S} 39]}$ & $\begin{array}{l}2014- \\
2015\end{array}$ & 1003 & 9.4 & $59.76 \pm 9.82$ & $9.24 \pm \mathrm{SD} ?$ & C3 \\
\hline 33 & $\begin{array}{l}\text { Al-Rubeaan, 2018, VH, Saudi } \\
\text { Arabia [S40] }\end{array}$ & $\begin{array}{l}2014- \\
2015\end{array}$ & 640 & 64.55 & $56.64 \pm 6.62$ & $18.21 \pm 5.93$ & $\mathrm{C} 1,2$ \\
\hline 34 & Heydari, 2010, H, Iran ${ }^{[\mathrm{S} 41]}$ & 2006 & 200 & 10 & $52.39 \pm 10.03$ & 0.5 & C3 \\
\hline 35 & Al-Futaisi, 2006, VH, Oman ${ }^{[\mathrm{S} 42]}$ & $\begin{array}{l}2002- \\
2003\end{array}$ & 261 & 27 & $50 \pm 13$ & NA & C3 \\
\hline 36 & Zakerkish, 2013, H, Iran [S43] & $\begin{array}{l}2010- \\
2011\end{array}$ & 350 & 25.7 & $54 \pm 10.5$ & $4.6 \pm 5.9$ & C3 \\
\hline 37 & Mansournia, 2017, H, Iran ${ }^{[S 44]}$ & $\begin{array}{l}2015- \\
2016\end{array}$ & 260 & 41.2 & $62.5 \pm 10.9$ & $10.8 \pm 8.9$ & $\mathrm{C} 3$ \\
\hline 38 & Khazai, 2006, H, Iran ${ }^{[S 45]}$ & 2002 & 200 & 3 & $48.58 \pm \mathrm{SD} ?$ & NA & NA \\
\hline
\end{tabular}

Codes [1]: VH (very high); H (high); M (medium); L (low).

Codes [2]: C1 (Urinary albumin-to-creatinine ratio); C2 (Sustained reduction in eGFR); C3 (Persistent albuminuria in $24 \mathrm{hr}$ urine); C4 (Semi-quantitative or qualitative). 


\section{Identification of studies via databases and registers}

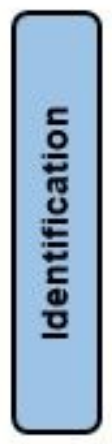

\begin{tabular}{|c|c|}
\hline $\begin{array}{l}\text { Records identified from*: } \\
\text { Databases }(n=3332) \\
\text { Registers }(n=0)\end{array}$ & $\begin{array}{l}\text { Records removed before screening: } \\
\text { Duplicate records removed ( } \mathrm{n}= \\
346 \text { ) } \\
\text { Records marked as ineligible by } \\
\text { automation tools ( } \mathrm{n}=0 \text { (not } \\
\text { used)) } \\
\text { Records removed for other } \\
\text { reasons }(n=43 \text { ) }\end{array}$ \\
\hline
\end{tabular}
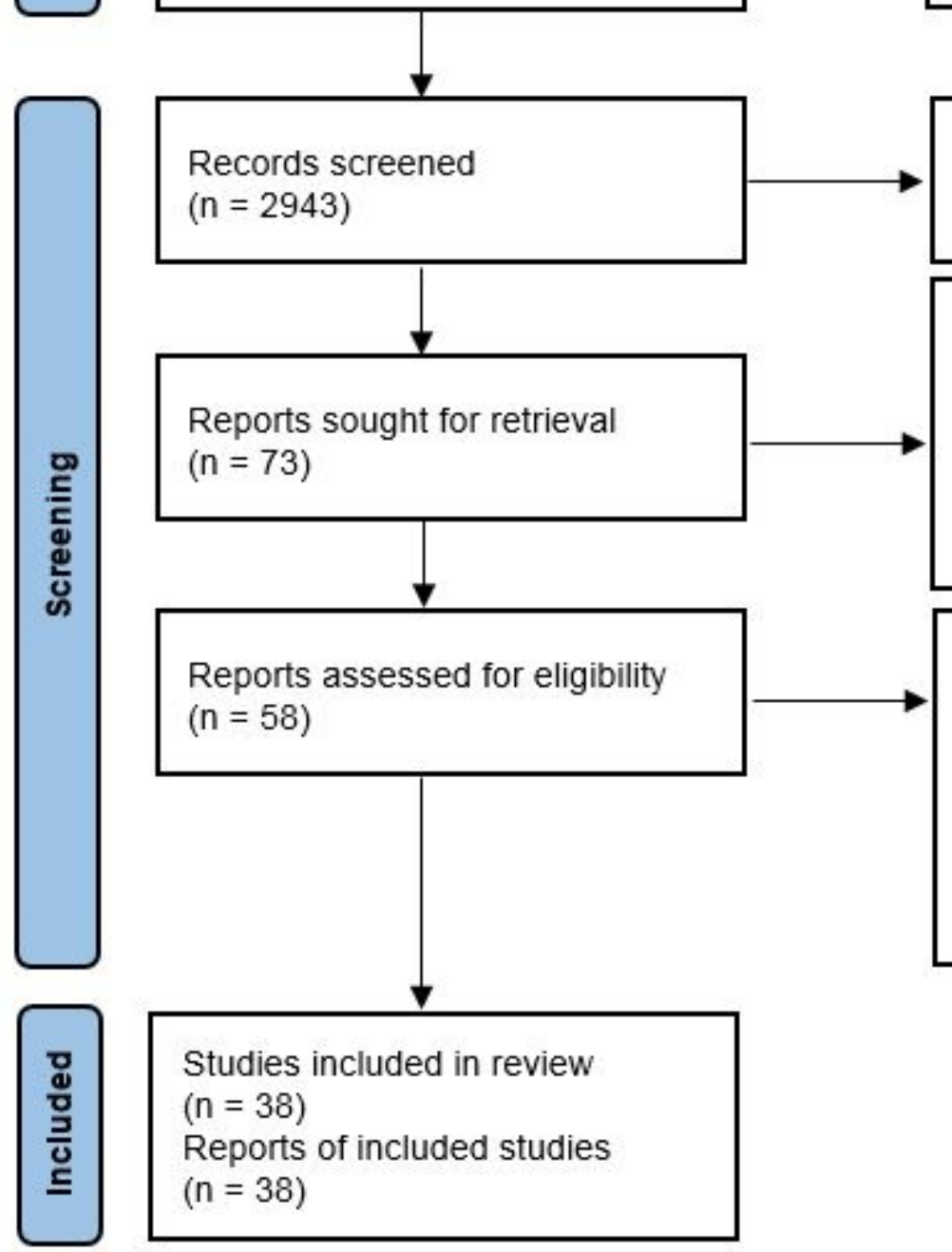

Records excluded ${ }^{* *}$

$(n=2870)$

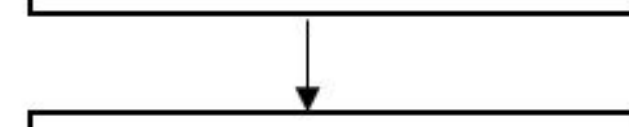

\section{Reports not retrieved}

$(n=15)$

Review or meta-analysis study (n

Reports sought for retrieval

$(n=73)$

$=5$ )

Non-English papers $(n=5)$

Full text unavailable $(n=5)$

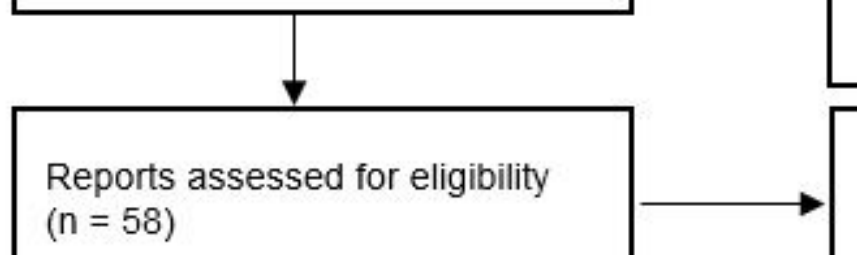

Reports excluded:

Studies out of EMRO $(n=4)$

Reporting incidence of

nephropathy $(n=2)$

Unclear results or irrelevant

papers $(n=14)$

Studies included in review

$(\mathrm{n}=38)$

Reports of included studies

$(n=38)$

\section{Figure 1}

Flowchart of search and screening result (PRISMA-2020-Flow-Diagram) 


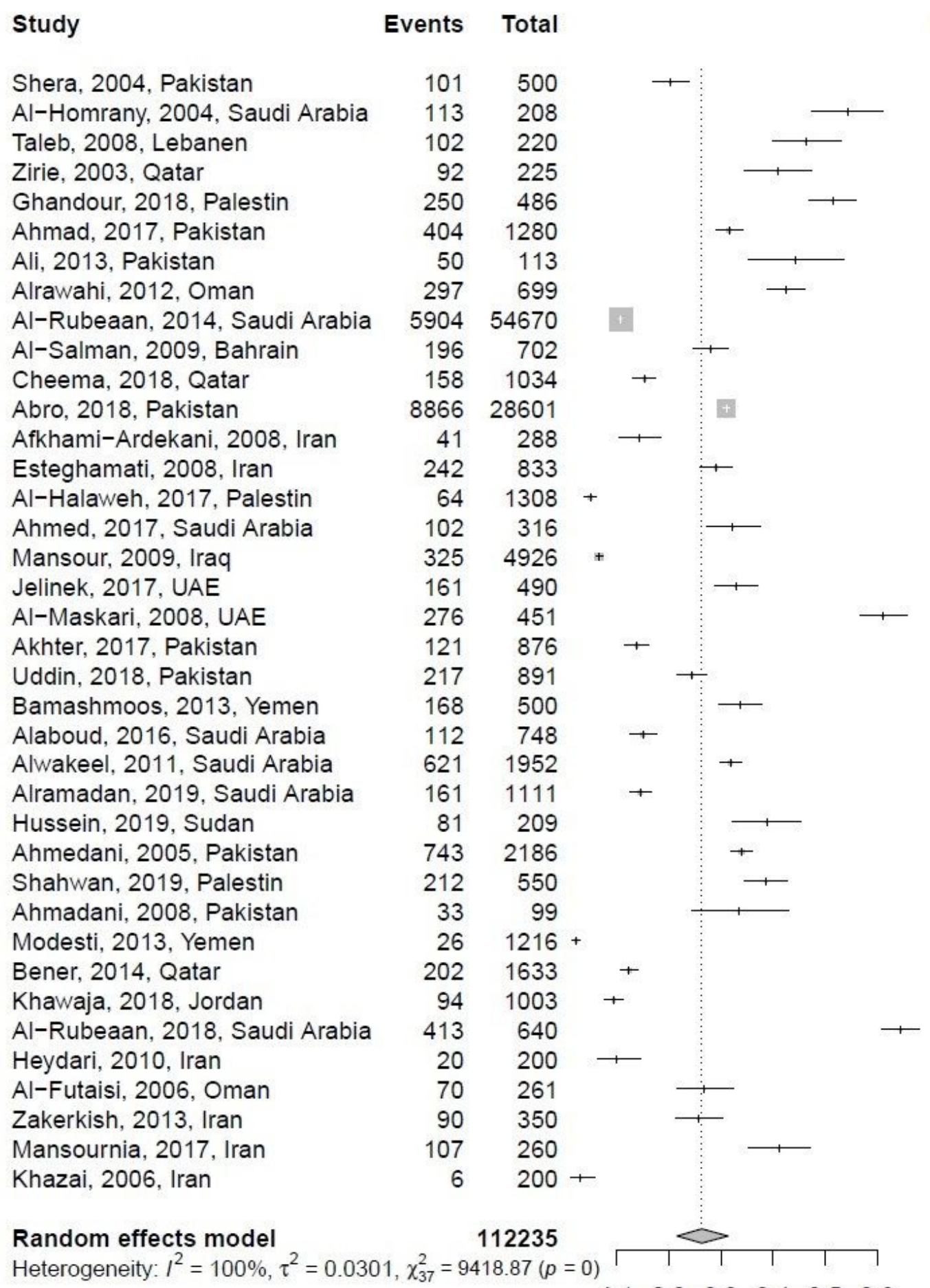

$\begin{array}{llllll}0.1 & 0.2 & 0.3 & 0.4 & 0.5 & 0.6\end{array}$

Prevalence of Nephropathy
Weight

Proportion $\quad 95 \%-\mathrm{Cl}$ (random)

$0.20[0.17 ; 0.24] 2.6 \%$

$0.54[0.48 ; 0.61] 2.6 \%$

$0.46[0.40 ; 0.53] \quad 2.6 \%$

$0.41[0.35 ; 0.47] \quad 2.6 \%$

$0.51[0.47 ; 0.56] \quad 2.6 \%$

$0.32[0.29 ; 0.34] 2.7 \%$

$0.44[0.35 ; 0.54] 2.5 \%$

$0.42[0.39 ; 0.46] \quad 2.7 \%$

$0.11[0.11 ; 0.11] \quad 2.7 \%$

$0.28[0.25 ; 0.31] 2.7 \%$

$0.15[0.13 ; 0.18] 2.7 \%$

$0.31[0.30 ; 0.32] \quad 2.7 \%$

$0.14[0.10 ; 0.19] \quad 2.6 \%$

$0.29[0.26 ; 0.32] \quad 2.7 \%$

$0.05[0.04 ; 0.06] \quad 2.7 \%$

$0.32[0.27 ; 0.38] \quad 2.6 \%$

$0.07[0.06 ; 0.07] \quad 2.7 \%$

$0.33[0.29 ; 0.37] \quad 2.6 \%$

$0.61[0.57 ; 0.66] 2.6 \%$

$0.14[0.12 ; 0.16] \quad 2.7 \%$

$0.24[0.22 ; 0.27] \quad 2.7 \%$

$0.34[0.30 ; 0.38] \quad 2.6 \%$

$0.15[0.13 ; 0.18] \quad 2.7 \%$

$0.32[0.30 ; 0.34] \quad 2.7 \%$

$0.14[0.12 ; 0.17] \quad 2.7 \%$

$0.39[0.32 ; 0.45] \quad 2.6 \%$

$0.34[0.32 ; 0.36] \quad 2.7 \%$

$0.39[0.35 ; 0.43] \quad 2.6 \%$

$0.33[0.24 ; 0.43] \quad 2.5 \%$

$0.02[0.01 ; 0.03] \quad 2.7 \%$

$0.12[0.11 ; 0.14] \quad 2.7 \%$

$0.09[0.08 ; 0.11] 2.7 \%$

$0.65[0.61 ; 0.68] 2.6 \%$

$0.10[0.06 ; 0.15] \quad 2.6 \%$

$0.27[0.22 ; 0.32] \quad 2.6 \%$

$0.26[0.21 ; 0.30] 2.6 \%$

$0.41[0.35 ; 0.47] 2.6 \%$

$0.03[0.01 ; 0.06] 2.6 \%$

$0.26[0.22 ; 0.31] 100.0 \%$

\section{Figure 2}

Forest plot of 38 studies 
Events Total

Abro, 2018, Pakistan

Al-Rubeaan, 2014, Saudi Arabia

Al-Rubeaan, 2018, Saudi Arabia

Ahmedani, 2005, Pakistan

Ahmad, 2017, Pakistan

Mansour, 2009, Iraq

Ghandour, 2018, Palestin

Esteghamati, 2008, Iran

Al-Salman, 2009, Bahrain

Alramadan, 2019, Saudi Arabia

Shahwan, 2019, Palestin

Bener, 2014, Qatar

Zakerkish, 2013, Iran

Zirie, 2003, Qatar

Bamashmoos, 2013, Yemen

Akhter, 2017, Pakistan

Ahmed, 2017, Saudi Arabia

Mansournia, 2017, Iran

Cheema, 2018, Qatar

Afkhami-Ardekani, 2008, Iran

Hussein, 2019, Sudan

Ahmadani, 2008, Pakistan

Khazai, 2006, Iran

375812569

238424328

$221 \quad 355$

$184 \quad 949$

$178 \quad 2513$

$171 \quad 334$

$130 \quad 472$

$127 \quad 490$

$103 \quad 724$

$97 \quad 249$

82791

59238

$59 \quad 151$

$48 \quad 280$

$42 \quad 422$$$
39
$$

\section{5}

15

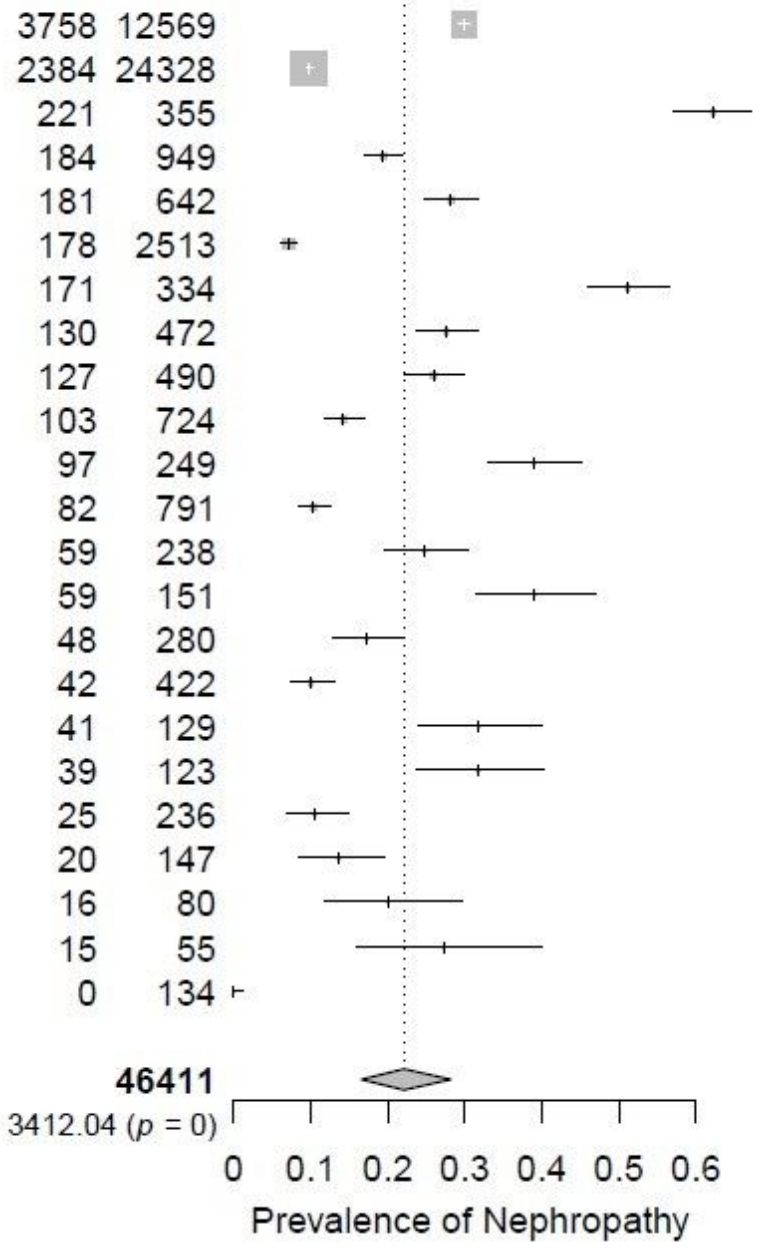

$181 \quad 642$

Random effects model

Heterogeneity: $I^{2}=99 \%, \tau^{2}=0.0282, \chi_{22}^{2}=3412.04(p=0)$

Prevalence of Nephropathy
Weight

Proportion $\quad 95 \%-\mathrm{Cl}$ (random)

$0.30[0.29 ; 0.31] \quad 4.5 \%$

$0.10[0.09 ; 0.10] \quad 4.5 \%$

$0.62[0.57 ; 0.67] \quad 4.4 \%$

$0.19[0.17 ; 0.22] \quad 4.5 \%$

$0.28[0.25 ; 0.32] \quad 4.5 \%$

$0.07[0.06 ; 0.08] \quad 4.5 \%$

$0.51[0.46 ; 0.57] \quad 4.4 \%$

$0.28[0.24 ; 0.32] \quad 4.4 \%$

$0.26[0.22 ; 0.30] \quad 4.4 \%$

$0.14[0.12 ; 0.17] \quad 4.5 \%$

$0.39[0.33 ; 0.45] \quad 4.4 \%$

$0.10[0.08 ; 0.13] \quad 4.5 \%$

$0.25[0.19 ; 0.30] \quad 4.4 \%$

$0.39[0.31 ; 0.47] \quad 4.3 \%$

$0.17[0.13 ; 0.22] \quad 4.4 \%$

$0.10[0.07 ; 0.13] \quad 4.4 \%$

$0.32[0.24 ; 0.40] \quad 4.2 \%$

$0.32[0.24 ; 0.40] \quad 4.2 \%$

$0.11[0.07 ; 0.15] \quad 4.3 \%$

$0.14[0.08 ; 0.20] \quad 4.3 \%$

$0.20[0.12 ; 0.30] \quad 4.1 \%$

$0.27[0.16 ; 0.40] \quad 3.9 \%$

$0.00[0.00 ; 0.01] \quad 4.2 \%$

$0.22[0.17 ; 0.28] 100.0 \%$

\section{Figure 3}

Forest plot of prevalence of nephropathy in women with type two diabetes 
Study

Abro, 2018, Pakistan

Al-Rubeaan, 2014, Saudi Arabia

Al-Rubeaan, 2018, Saudi Arabia

Ahmedani, 2005, Pakistan

Ahmad, 2017, Pakistan

Mansour, 2009, Iraq

Ghandour, 2018, Palestin

Esteghamati, 2008, Iran

Al-Salman, 2009, Bahrain

Alramadan, 2019, Saudi Arabia

Shahwan, 2019, Palestin

Bener, 2014, Qatar

Zakerkish, 2013, Iran

Zirie, 2003, Qatar

Bamashmoos, 2013, Yemen

Akhter, 2017, Pakistan

Ahmed, 2017, Saudi Arabia

Mansournia, 2017, Iran

Cheema, 2018, Qatar

Afkhami-Ardekani, 2008, Iran

Hussein, 2019, Sudan

Ahmadani, 2008, Pakistan

Khazai, 2006, Iran

Random effects model

Heterogeneity: $I^{2}=99 \%, \tau^{2}=0.0314, \chi_{22}^{2}=4279.79(p=0)$

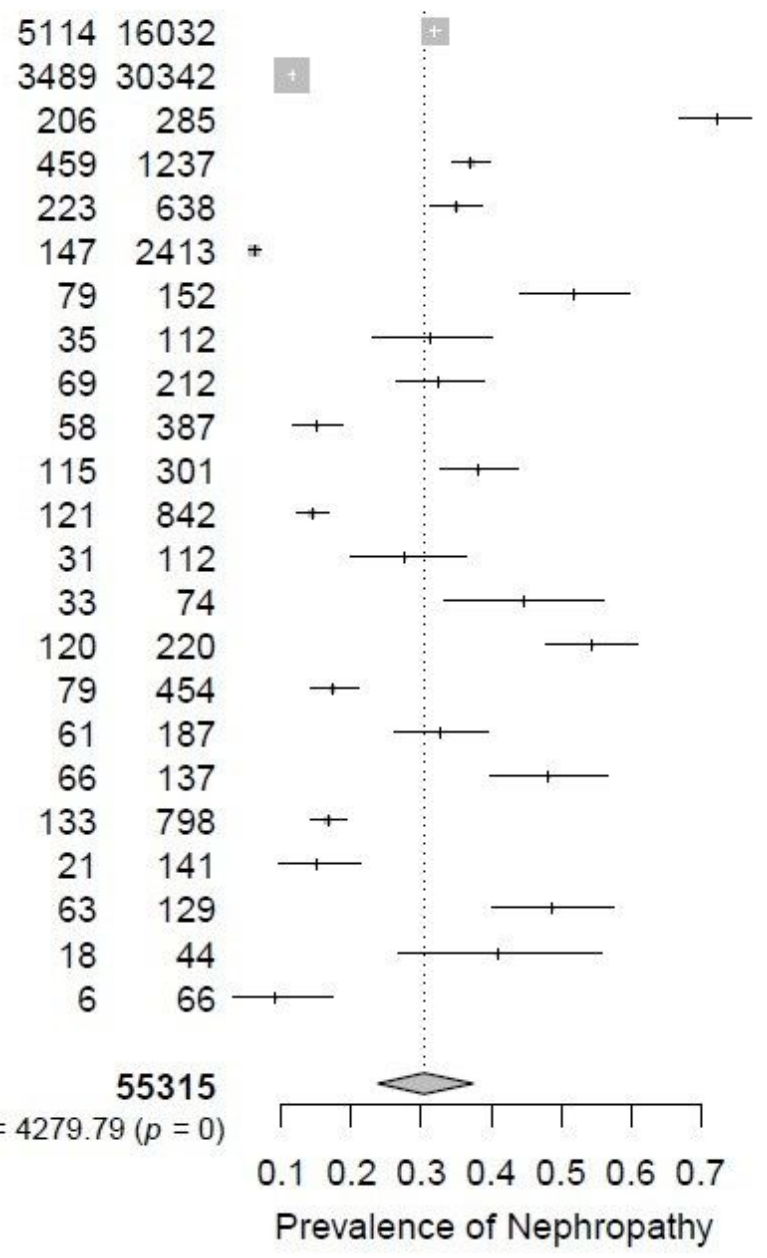

Weight

Proportion

$95 \%-\mathrm{Cl}$ (random)

$0.32[0.31 ; 0.33] \quad 4.5 \%$

$0.11[0.11 ; 0.12] \quad 4.5 \%$

$0.72[0.67 ; 0.77] \quad 4.4 \%$

$0.37[0.34 ; 0.40] \quad 4.5 \%$

$0.35[0.31 ; 0.39] \quad 4.5 \%$

$0.06[0.05 ; 0.07] \quad 4.5 \%$

$0.52[0.44 ; 0.60] \quad 4.3 \%$

$0.31[0.23 ; 0.40] \quad 4.2 \%$

$0.33[0.26 ; 0.39] \quad 4.4 \%$

$0.15[0.12 ; 0.19] \quad 4.4 \%$

$0.38[0.33 ; 0.44] \quad 4.4 \%$

$0.14[0.12 ; 0.17] \quad 4.5 \%$

$0.28[0.20 ; 0.36] \quad 4.2 \%$

$0.45[0.33 ; 0.56] \quad 4.1 \%$

$0.55[0.48 ; 0.61] \quad 4.4 \%$

$0.17[0.14 ; 0.21] \quad 4.5 \%$

$0.33[0.26 ; 0.40] \quad 4.3 \%$

$0.48[0.40 ; 0.57] \quad 4.3 \%$

$0.17[0.14 ; 0.19] \quad 4.5 \%$

$0.15[0.09 ; 0.21] \quad 4.3 \%$

$0.49[0.40 ; 0.57] \quad 4.3 \%$

$0.41[0.27 ; 0.56] \quad 3.8 \%$

$0.09[0.03 ; 0.17] \quad 4.0 \%$

$0.30[0.24 ; 0.37] 100.0 \%$

\section{Figure 4}

Forest plot of prevalence of nephropathy in men with type two diabetes 

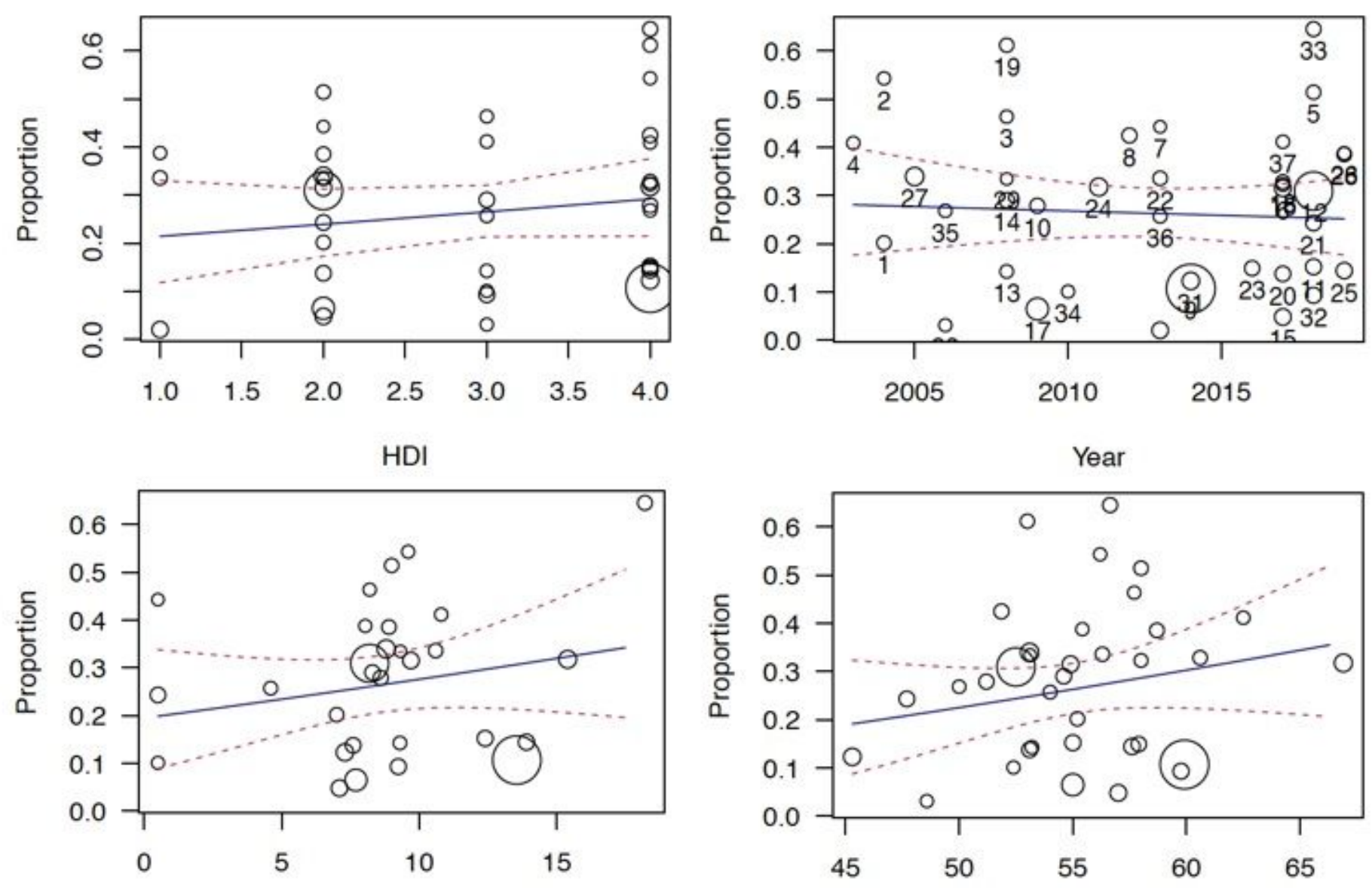

Mean duration of diagnosed type 2 diabetes

Mean Age

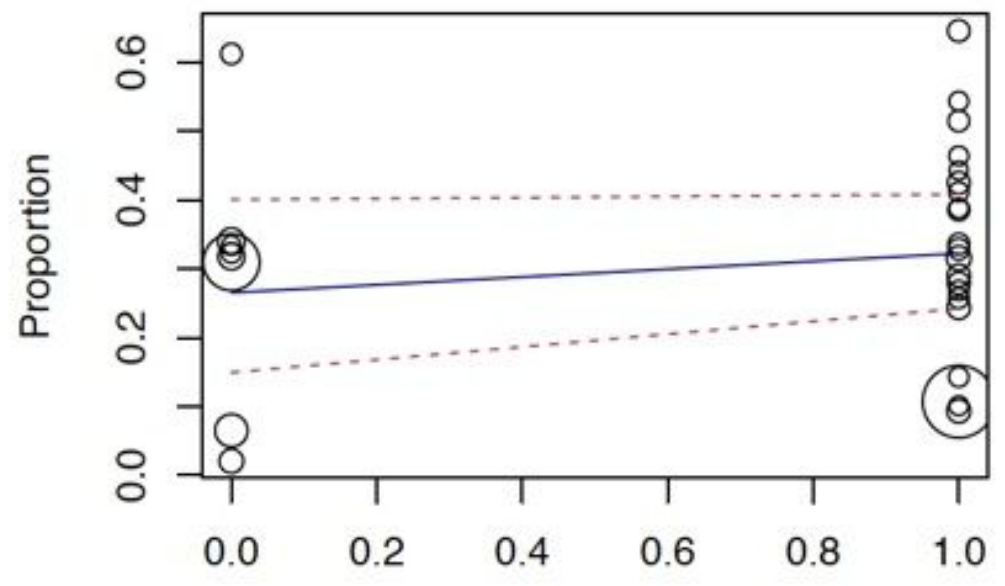

Diagnostic Test

\section{Figure 5}

The scatter plot of (A) HDI, (B) publication year, (C) mean duration of diagnosed diabetes, (D) mean age, and $(E)$ diagnostic test by the effect sizes [Note for interpretation: 1- Each study represented by a circle that its size was proportional to the study size; 2 - in fig 5-E, 0 denotes studies that used semi-quantitative or qualitative diagnostic tests like Micral test or Dipstick and 1 denotes studies that used qualitative diagnostic tests] 


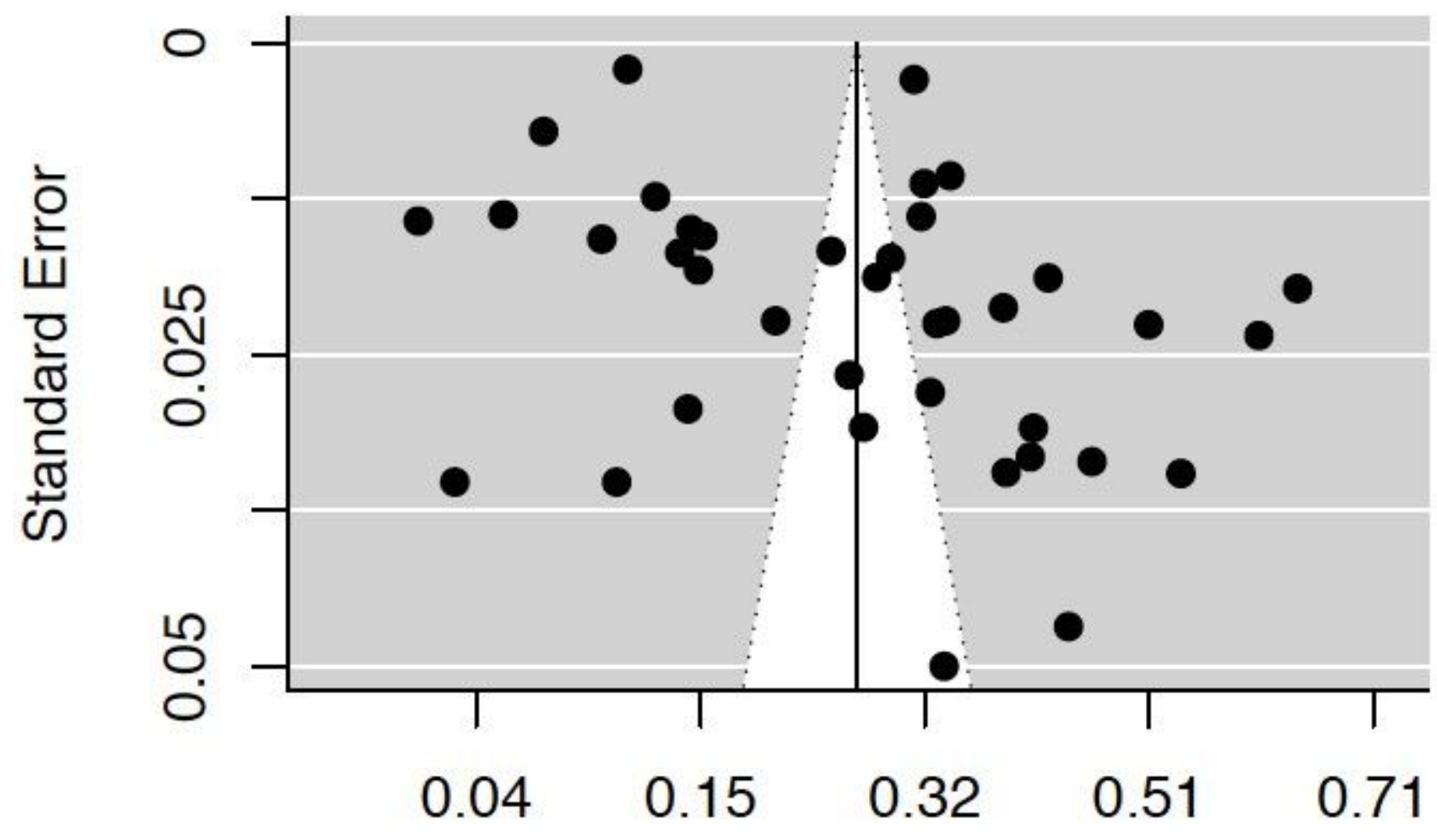

\section{Proportion}

Figure 6

The funnel plot of effect size against standard error [Note for interpretation: Each dot denotes a study; the vertical line denotes the summary effect size; two limit lines denotes $95 \% \mathrm{Cl}$ of the summary effect size.]

\section{Supplementary Files}

This is a list of supplementary files associated with this preprint. Click to download.

- Appendix1.docx

- Appendix2.docx

- Appendix3.docx

- Appendix4.docx

- Appendix5.docx

- Appendix6.docx

- Appendix7.docx

- Appendix8.docx

- Appendix9.docx

- Appendix10.docx 
- Supplementaryreferences.docx 